TecnoLógicas

ISSN-p 0123-7799

ISSN-e 2256-5337

Vol. 22, No. 46, pp 25-38

Sep-dic de 2019

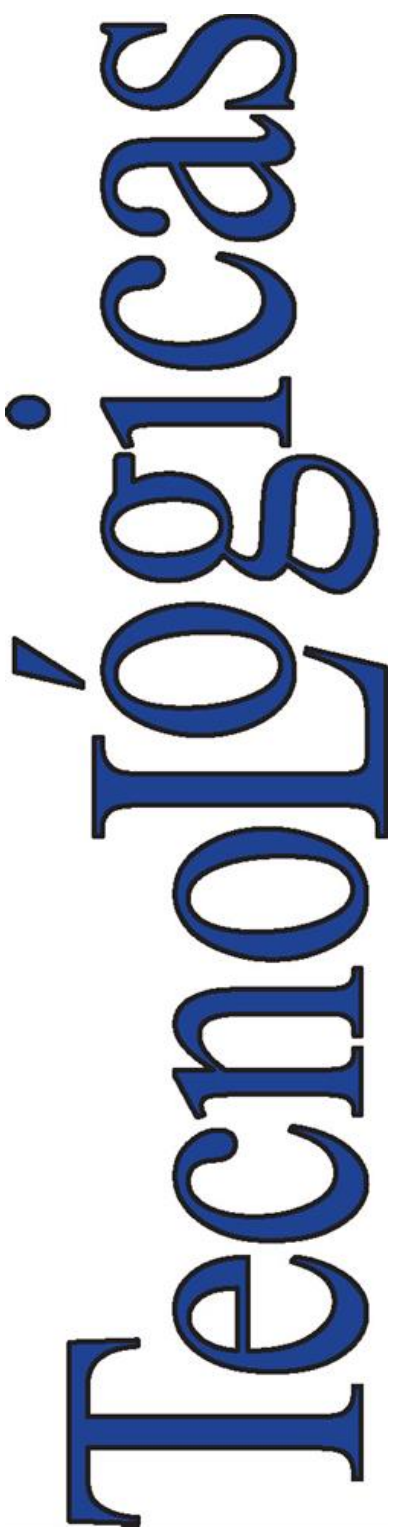

(C) Instituto Tecnológico Metropolitano Este trabajo está licenciado bajo una Licencia Internacional Creative Commons Atribución (CC BY-NC-SA)

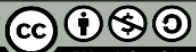

Artículo de Investigación/Research Article

\section{Evaluación de los parámetros del proceso de congelación para la pulpa de Açaí}

\author{
Parameters for Açaí Pulp Freezing \\ Sebastián Arias-Giraldo ${ }^{1}$, Adela M. Ceballos- \\ Peñaloza (D)2 y Luis F. Gutiérrez-Mosquera (D) ${ }^{3}$
}

Recibido: 23 de noviembre de 2018

Aceptado: 06 de junio de 2019

Cómo citar / How to cite

S. Arias-Giraldo, A. M. Ceballos-Peñaloza y L. F. Gutiérrez-Mosquera "Evaluación de los parámetros del proceso de congelación para la pulpa de Açaí”. TecnoLógicas, vol. 22, no. 46, pp. 25-38, 2019.

https://doi.org//10.22430/22565337.1117
1 M.Sc. en Ingeniería de Alimentos, Facultad de Ciencias Administrativas, Económicas y Contables, Grupo de Investigación Goras, Universidad Católica Luis Amigó, Medellín-Colombia, sebastian.ariasgi@amigo.edu.co

2 M.Sc. Ingeniería Química, Grupo de Investigación Alimentos y Agroindustria, Universidad de Caldas, Manizales-Colombia, adela.ceballos@ucaldas.edu.co

3 Ph.D. Ingeniería, Facultad de Ingeniería, Grupo de Investigación Alimentos y Agroindustria, Universidad de Caldas, Manizales-Colombia, fernando.gutierrez@ucaldas.edu.co 


\title{
Resumen
}

La refrigeración y la congelación son procesos tradicionalmente utilizados en la conservación de alimentos. En el caso del açaí, una fruta brasilera, estas tecnologías corresponden a los métodos más empleados por la industria. La aplicación de modelos teóricos para la predicción de tiempos y temperaturas de congelación permite mantener un control eficaz de dicha operación. El objetivo de este trabajo fue estimar y evaluar el tiempo, temperatura y velocidad de dos procesos de congelación para pulpa de açaí, tanto convencional como criogénico. El tiempo de congelación convencional para el producto fue $153,68 \pm 6,42 \mathrm{~min}$, con mínimo error respecto a las ecuaciones de Pham $(0,54 \%)$ y Nagaoka (1,71\%). Para la congelación rápida, el tiempo fue de 100,56 $\pm 17,90 \mathrm{~s}(1,68 \pm 0,29 \mathrm{~min})$, siendo representado eficazmente por el modelo de Nagaoka (6,81\%). Las temperaturas de inicio de la congelación fueron, para la operación lenta y rápida, de $-0,64 \pm 0,02{ }^{\circ} \mathrm{C}$ y $-2,91 \pm$ $0,86{ }^{\circ} \mathrm{C}$. Por medio de estos resultados, es posible obtener y validar correlaciones que permitan predecir efectivamente los tiempos de congelación de la pulpa de açaí fresca, al considerar los principales parámetros del proceso: geometría, temperatura del medio frio y composición del alimento.

\section{Palabras clave}

Açaí, congelación criogénica, Euterpe olerácea, ingeniería de alimentos.

\begin{abstract}
Refrigeration and freezing have been traditionally used in food preservation. In the industry of açaí, a Brazilian fruit, they are the most common technologies. The application of theoretical models for predicting freezing times and temperatures allows an effective control of those operations. The objective of this work was to evaluate the time, temperature, and speed of two processes, conventional and cryogenic freezing, for açaí pulp. The conventional freezing time for said product was $153.68 \pm 6.42 \mathrm{~min}$, with a minimal error with respect to Pham's (0.54 \%) and Nagaoka's (1.71\%) equations. The fast freezing time, in turn, was $100.56 \pm 17.90 \mathrm{~s}(1.68 \pm 0.29 \mathrm{~min})$, which is efficiently represented by Nagaoka's equation $(6,81 \%)$. The initial freezing temperatures for the slow and fast modes were -0.64 $\pm 0.02{ }^{\circ} \mathrm{C}$ and $-2.91 \pm 0.86{ }^{\circ} \mathrm{C}$, respectively. Based on these results, correlations can be obtained and validated to effectively predict the freezing times of fresh açaí pulp considering the main parameters in the process: geometry, cold medium temperature, and food composition.
\end{abstract}

\section{Keywords}

Açaí, cryogenic freezing, Euterpe oleracea, food engineering. 


\section{INTRODUCCIÓN}

El açaí es el fruto del açaizeiro (Euterpe olerácea), palmera típica brasilera que crece en los estados de Pará, Maranhão y Amazonas; cuya cadena productiva presenta un creciente interés, pasando del consumo local a la conquista de mercados de exportación. En el año 2017, la producción total del fruto en Brasil fue de 219885 toneladas, movilizando cifras económicas de 596,8 millones de reales [1].

La pulpa del açaí es muy apreciada por sus características nutritivas y sensoriales, siendo considerada como un alimento de alto valor calórico, buena fuente de vitamina $\mathrm{E}$, fibra, manganeso, cobre, calcio, magnesio y potasio [2]. Es considerado un alimento funcional gracias a su elevado contenido de antocianinas, incluyendo efecto anti-cancerígeno y antiinflamatorio, previniendo la aparición de enfermedades cardiovasculares y dolencias neurológicas [3], [4]. Además, el aceite del fruto tiene una composición de ácidos grasos de buena calidad, $60 \%$ mono insaturados y $13 \%$ poli insaturados [5].

En Brasil, el derivado más común del açaí es su pulpa, procesada hasta $72 \mathrm{~h}$ después de cosechado, permaneciendo durante este tiempo en condiciones de elevada humedad relativa y temperatura que ocasionan reacciones de oxidación, reducción del contenido de antocianinas y despigmentación [6], [7].

La refrigeración y la congelación son métodos convencionalmente usados para garantizar la vida útil de frutas $\mathrm{y}$ hortalizas. En la refrigeración se elimina el calor sensible y metabólico, reduciéndose la temperatura hasta un valor entre $4-7^{\circ} \mathrm{C}$. En la congelación, por el contrario, se elimina calor latente lográndose la transformación casi completa del agua en hielo, trabajándose a temperaturas medias de $-30{ }^{\circ} \mathrm{C}$ [8]. Según EMBRAPA [5], la congelación de la pulpa de açaí a temperaturas de $-20 \quad{ }^{\circ} \mathrm{C}$ inhibe significativamente la acción microbiana, y limita la actividad de las enzimas peroxidasa y polifenoloxidasa.

Según Barreiro y Sandoval [9], el tiempo nominal de congelación es el periodo necesario para que el centro térmico de un producto, que se encuentra en su temperatura inicial de congelación, alcance la temperatura final de congelación. El tiempo efectivo, por su parte, corresponde al tiempo total requerido para disminuir la temperatura desde cierto valor inicial por encima de su punto de congelación, hasta su temperatura final. La tasa media de congelamiento (o factor de penetración de calor) es el cociente entre la dimensión característica del producto y el tiempo nominal de congelación; mientras que la velocidad media es la razón entre la diferencia de las temperaturas inicial y final de congelación, y el tiempo usado para alcanzar dicho diferencial [10].

La calidad de un producto congelado depende de la temperatura y velocidad de operación, pues determinan la distribución y el tamaño de los cristales de hielo formados en los tejidos [11]. Cuando el proceso es lento, se favorece la formación de grandes masas de hielo que aumentan los espacios extracelulares y la presión osmótica. La acción mecánica de estos cristales provocan el deterioro de la textura, y originan la pérdida de líquidos durante la descongelación [12]. En la congelación rápida, la cristalización ocurre de forma simultánea en los espacios internos y extracelulares, siendo mínimo el deterioro del producto en comparación con el proceso convencional. Cuando se emplean temperaturas menores a $-70^{\circ} \mathrm{C}$ se congela agua ligada en el alimento, obteniéndose una solidificación cercana al $100 \%$ del agua del producto [13]-[15].

Desde el punto de vista termodinámico, el cambio de fase en la congelación de alimentos ocurre a temperatura variable, entre la temperatura inicial de congelación y la temperatura de congelación final o técnica [11], [16]. En el punto inicial de 
congelación solo aparecen cristales de hielo puro. A medida que avanza el proceso, aumenta la concentración de la solución y la formación de cristales de agua se da a temperaturas menores, de forma correspondiente con las propiedades coligativas de las soluciones [17].

El presente trabajo tuvo como objetivo determinar los tiempos teóricos $\mathrm{y}$ experimentales de congelación para pulpa de açaí bajo dos esquemas tecnológicos, empleando un congelador convencional de aire estático y una inmersión en nitrógeno líquido. Se definieron algunas variables esenciales en la conservación por frío: temperatura inicial y final de congelación, fracción de agua congelada y cinéticas de congelación del producto, para cada velocidad de proceso evaluada.

Cada sistema, criogénico y convencional, se estudió de manera independiente, ya que, al responder a diversos principios físicos $\mathrm{y}$ termodinámicos, se espera encontrar una respuesta cinética diferente para cada tecnología. Ambos métodos son ampliamente utilizados por el sector de frutas y hortalizas. Por medio de la investigación, se buscó seleccionar el mejor modelo matemático para el diseño del proceso de conservación por frío, tanto lento como rápido; sugiriendo, además, temperaturas iniciales $y$ finales de congelación, de manera que la pulpa de açaí no se someta a un tratamiento excesivo que deteriore sus atributos nutricionales y/o sensoriales.

\section{METODOLOGÍA}

\subsection{Materia prima}

Los experimentos se desarrollaron en los Laboratorios de Calidad y Seguridad Alimentaria del Departamento de Tecnología de Alimentos de la Universidad Federal de Vicosa, ubicada en Minas
Gerais, Brasil. 3 lotes de pulpa de açaí, de $2 \mathrm{~kg}$ cada uno, se adquirieron en la ciudad de Belém, estado de Pará. El producto se mantuvo en congelación a $-18{ }^{\circ} \mathrm{C}$ y, un día antes de los análisis, se descongeló bajo refrigeración a $7{ }^{\circ} \mathrm{C}$. Posteriormente se llevó a temperatura ambiente para los ensayos experimentales.

\subsection{Equipo de congelación}

Se construyó una caja de aluminio de 7 $\mathrm{cm} \times 7 \mathrm{~cm} \times 4 \mathrm{~cm}$, donde se colocaron $187 \mathrm{~g}$ de pulpa de açaí en cada experimento, de forma que se mantuviera una geometría de placa con espesor constante $(4 \mathrm{~cm})$. Se fijó un termopar (Marca TESTO, modelo AG 1006) para hacer la lectura de las temperaturas en el punto frío. Otro termopar se utilizó para conocer la temperatura del medio refrigerante.

Se realizó un proceso de congelación rápido y otro lento. Para el proceso lento, se puso en contacto la superficie de la placa con el aire frío. En la congelación rápida, se utilizó una caja de icopor con tapa como reservorio de nitrógeno. La caja metálica se aseguró a la tapa del contenedor por medio de tornillos, y los termopares se fijaron a dicha placa. El recipiente se cerró al iniciar el proceso como en la Fig. 1.

\subsection{Cinética de congelación}

Los termopares se integraron a un software computacional, que permitió la toma de datos cada 3 s. Se hizo el seguimiento de la cinética de congelación, y se construyeron las curvas de proceso (temperatura en el punto frío vs. tiempo) para cada sistema. Las temperaturas promedio de los medios de enfriamiento fueron $-22{ }^{\circ} \mathrm{C}$ y $-196{ }^{\circ} \mathrm{C}$, en el esquema convencional y criogénico, respectivamente. 

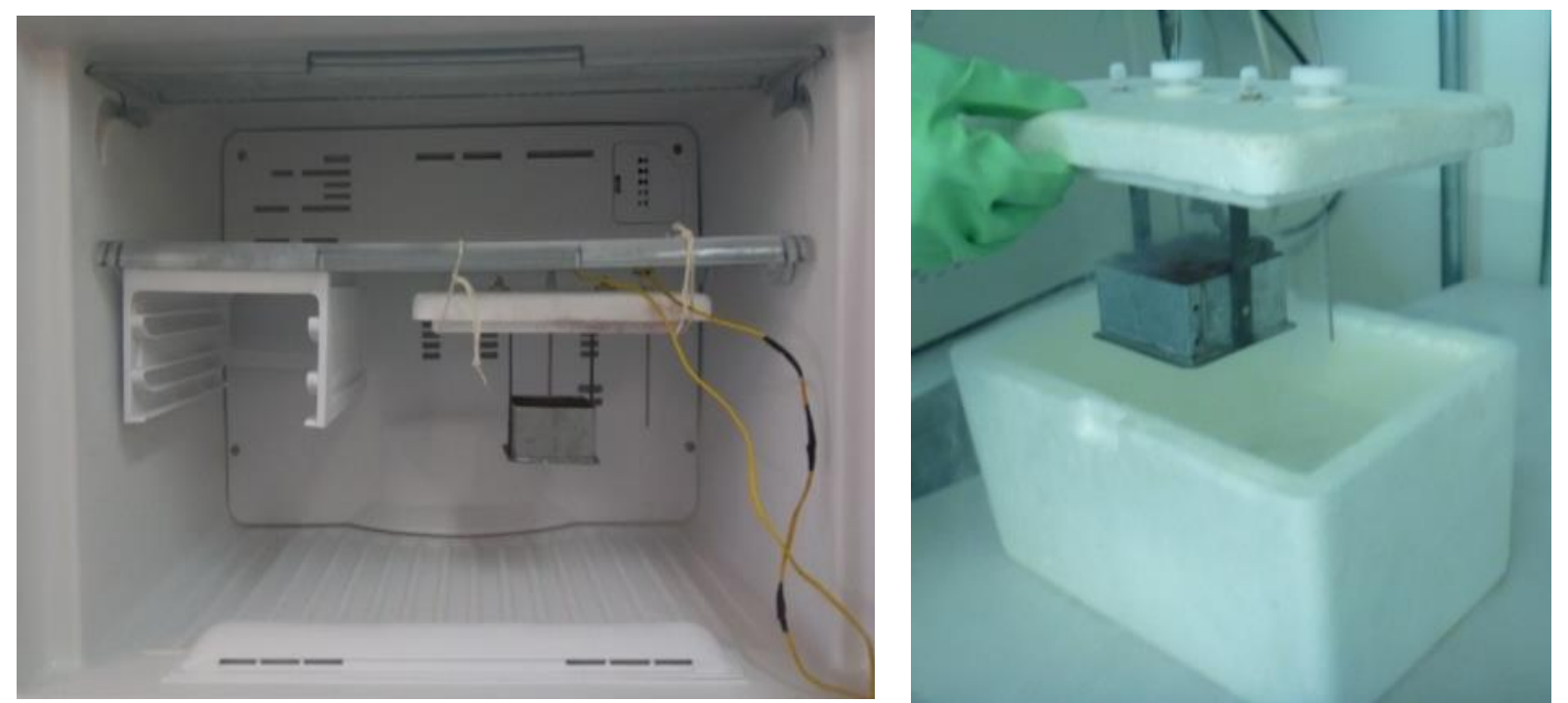

Fig. 1. Montajes experimentales para congelación lenta (izquierda) y rápida (derecha). Fuente: autores.

\subsection{Determinación experimental de los parámetros de operación}

Para cada curva de proceso, se encontraron las temperaturas inicial $\mathrm{y}$ final de congelación de la pulpa. Los resultados se obtuvieron mediante ajuste a una tendencia lineal de los puntos, entre el primer cambio de pendiente en la curva y la porción de datos posteriores que ofrecieran mayor cercanía al modelo lineal.

El tiempo de congelación real se estimó con una diferencia entre el tiempo inicial y final de proceso. Las tasas y velocidades se determinaron según Barreiro y Sandoval [9] y Cháves, et al. [10].

\subsection{Cálculo de tiempo de enfriamiento}

Para el cálculo del tiempo de enfriamiento se utilizaron ecuaciones de transferencia de calor en estado transitorio, y los diagramas termodinámicos clásicos propuestos por Gurney y Lurie [18] para placas.

$$
\begin{gathered}
\theta=\frac{\Delta t}{\Delta t_{0}}=\frac{T-T_{a}}{T_{0}-T_{a}} \\
\mathrm{~B}_{\mathrm{i}}=\frac{h \cdot D}{k}
\end{gathered}
$$

$$
\begin{gathered}
\mathrm{F}_{\mathrm{o}}=\frac{k}{\rho \cdot C_{p}} \frac{t}{D^{2}} \\
\mathrm{~m}=\frac{1}{B i}
\end{gathered}
$$

Las ecuaciones (1), (2), (3) y (4), fueron tomadas de Orrego [19], donde $T$ es la temperatura final de enfriamiento $\left({ }^{\circ} \mathrm{C}\right), T_{0}$ la temperatura inicial del producto $\left({ }^{\circ} \mathrm{C}\right), T_{a}$ la temperatura del medio frío $\left({ }^{\circ} \mathrm{C}\right), h$ el coeficiente convectivo de calor $\left(\mathrm{W} / \mathrm{m}^{2} .{ }^{\circ} \mathrm{C}\right), D$ la dimensión característica $(\mathrm{m}), k$ la conductividad térmica del alimento $\left(\mathrm{W} / \mathrm{m} .{ }^{\circ} \mathrm{C}\right), \quad \mathrm{B}_{\mathrm{i}}$ el número de Biot, $\rho$ la densidad del alimento $\left(\mathrm{kg} / \mathrm{m}^{3}\right), C_{p}$ el calor específico del alimento $\left(\mathrm{kJ} / \mathrm{kg} .{ }^{\circ} \mathrm{C}\right), \quad t$ el tiempo de enfriamiento ( $\mathrm{s}$ ), $\mathrm{F}_{\mathrm{o}}$ el número de Fourier, y m es un factor de forma.

Para predecir los tiempos de proceso se emplearon valores del coeficiente convectivo reportados por Erickson y Hung [13], para una congelación convencional y criogénica respectivamente: $\mathrm{h}=10$ $\mathrm{W} / \mathrm{m}^{2} .{ }^{\circ} \mathrm{C}$ y $\mathrm{h}=1.500 \mathrm{~W} / \mathrm{m}^{2} .{ }^{\circ} \mathrm{C}$.

Los valores para las propiedades termofísicas de la pulpa, utilizadas en el desarrollo de los cálculos de ingeniería, se tomaron del estudio publicado por Arias, et al. [20]. 


\subsection{Parámetros de congelación}

\subsubsection{Temperatura inicial de congelación}

Se calculó con base a la adaptación de la ley de Raoult, para productos alimenticios con humedad mayor a $80 \%$ [19]. Las ecuaciones (5) y (6) muestran el modelo utilizado. El factor $\mathrm{M}$ se determinó con la ecuación (7), propuesta por Chen [21] para jugos de naranja y manzana.

$$
\begin{gathered}
T c=\frac{-K w \cdot C}{M} \\
C=\frac{1-m_{w}}{m_{w}} \times 100 \\
M=\frac{200}{0,25+S}
\end{gathered}
$$

donde, $K w$ tiene un valor de 18,6 y es la constante criogénica del agua, $C$ es la masa de soluto disuelto en $100 \mathrm{~g}$ de agua, $m_{w}$ la fracción másica de agua en el producto, $M$ el peso molecular del soluto $(\mathrm{kg} / \mathrm{kmol})$ y $S$ la fracción de sólidos del alimento.

\subsubsection{Fracción de agua congelada (y)}

Las ecuaciones (8) y (9) presentan el modelo de Chen [21], utilizado en este trabajo para estimar teóricamente la cantidad de agua congelada.

$$
\begin{gathered}
G=\frac{S}{M}\left[\frac{R \cdot T_{0}^{2}}{L}\right]\left[\frac{\mathrm{T}_{\mathrm{c}}-\mathrm{T}}{\left(T-T_{0}\right)\left(T_{c}-T_{0}\right)}\right] \\
y=\frac{G}{m_{w}}
\end{gathered}
$$

$G$ es la fracción de hielo en el producto a congelar, $S$ el contenido de sólidos totales, $M$ el peso molecular del soluto $(\mathrm{kg} / \mathrm{kmol}), R$ $=8,32 \mathrm{~kJ} / \mathrm{kmol} . \mathrm{K}, L$ el calor latente de fusión del agua $(335 \mathrm{~kJ} / \mathrm{kg}), T_{0}$ la temperatura estándar de fusión del agua, $T_{c}$ la temperatura inicial de congelación (K), T la temperatura del sistema en cualquier momento (K), y la fracción de agua congelada en el alimento, y $m_{w}$ el contenido de agua sin congelar.

\subsubsection{Tiempo teórico de congelación}

La Tabla 1 muestra los modelos usados para predecir los tiempos de congelación de la pulpa de açaí.

\subsection{Diseño experimental}

La obtención de los parámetros del proceso de congelación, se organizó en un diseño experimental completamente aleatorio con tres lotes diferentes de producto. Los ensayos se realizaron por triplicado para cada lote.

Los resultados del estudio de la temperatura inicial de congelación y del análisis de los tiempos de proceso se interpretaron a través de un ANOVA, por medio de una prueba $\mathrm{F}$ con una significancia de $5 \%$. Para evaluar la diferencia entre las medias de tiempo y temperatura de operación de cada modelo, incluyendo el resultado real, se utilizó el test de Tukey con $\alpha=5 \%$.

Los análisis estadísticos se realizaron con el programa SAS versión 9.2 (Statistical Analysis System; SAS Institute Inc; USA), licenciado para la Universidad Federal de Viçosa.

\section{RESULTADOS Y DISCUSIÓN}

\subsection{Curvas de congelación}

Las Fig. 2 y Fig. 3 son un ejemplo de las curvas de congelación obtenidas en este trabajo, las cuales presentaron un comportamiento típico para un proceso en congelador convencional y con nitrógeno líquido, respectivamente [17]-[19].

En cada curva fue posible observar las tres fases de operación: el pre enfriamiento, la congelación y el enfriamiento posterior. 
Evaluación de los parámetros del proceso de congelación para la pulpa de Açaí

Tabla 1. Modelos matemáticos para la predicción de los tiempos de congelación*. Fuente: autores.

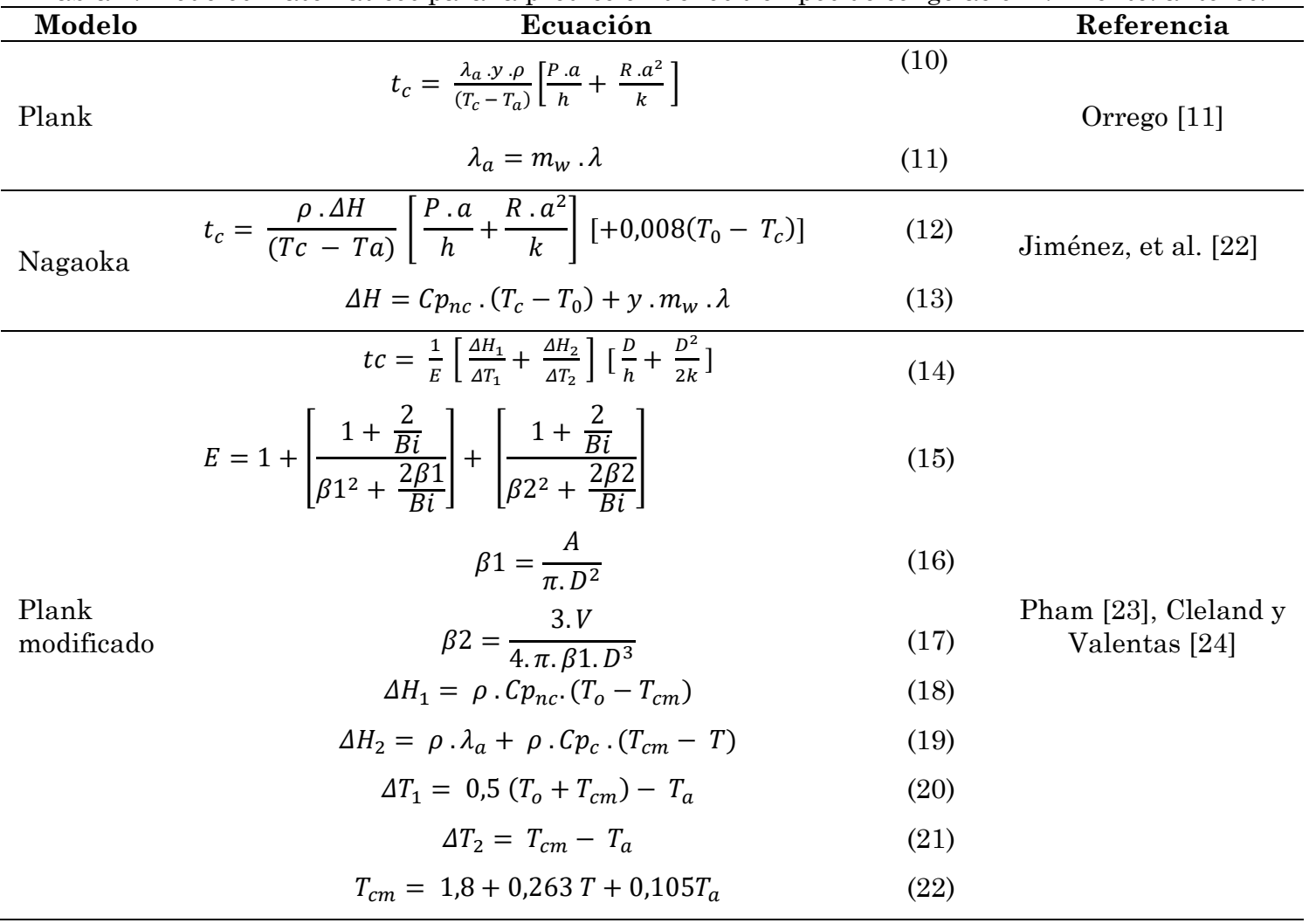

\footnotetext{
$* t_{c}$ es el tiempo de congelación del alimento (s), $\rho$ es la densidad del alimento sin congelar $\left(\mathrm{Kg} / \mathrm{m}^{3}\right), \lambda_{a}$ es el calor latente de congelación del alimento $(\mathrm{kJ} / \mathrm{Kg}), \lambda$ es el calor latente estándar de fusión del agua $(335 \mathrm{~kJ} / \mathrm{kg}), m_{w}$ es la fracción másica de agua en el alimento, $h$ es el coeficiente convectivo de transferencia de calor $\left(\mathrm{W} / \mathrm{m}^{2}{ }^{\circ} \mathrm{C}\right), k$ es la conductividad térmica del alimento congelado $\left(\mathrm{W} / \mathrm{m} .{ }^{\circ} \mathrm{C}\right), a$ es la dimensión característica del alimento $(\mathrm{m}), P$ y $R$ son las constantes de Plank, $T_{c}$ es la temperatura inicial de congelamiento $\left({ }^{\circ} \mathrm{C}\right)$ y $T_{a}$ es la temperatura del medio frío $\left({ }^{\circ} \mathrm{C}\right)$. Para conocer las constantes de Plank se usó el método gráfico propuesto por López-Leiva y Hallstrom [25]. En Nagaoka, $\Delta H$ es la diferencia de entalpía durante el enfriamiento y congelación del alimento (kJ/kg), $C p_{n c}$ es el calor específico sin congelar $\left(\mathrm{kJ} / \mathrm{kg} .{ }^{\circ} \mathrm{C}\right)$, $y$ es la fracción de agua congelada, y $T_{0}$ es la temperatura inicial del producto $\left({ }^{\circ} \mathrm{C}\right)$. Para el modelo de Pham, $E$ es un factor de forma que varía entre 1 y $3, \beta 1$ y $\beta 2$ son factores de forma, $A$ es el área seccional $\left(\mathrm{m}^{2}\right), D$ es el semi espesor $(\mathrm{m}), V$ es el volumen del alimento $\left(\mathrm{m}^{3}\right), T_{c m}$ es la temperatura media $\left({ }^{\circ} \mathrm{C}\right), C p_{c}$ es el calor específico congelado $\left(\mathrm{kJ} / \mathrm{kg} .{ }^{\circ} \mathrm{C}\right)$, y $T$ es la temperatura final de congelación $\left({ }^{\circ} \mathrm{C}\right)$.
}

En el caso convencional, se pudo notar de forma contundente el cambio de fase del agua y el gradiente de temperatura en que se dio la congelación. En el congelamiento criogénico también se observó el paso del açaí por los estadios de congelación; sin embargo, estos cambios no se apreciaron con exactitud pues el choque térmico fue muy grande.

En la operación lenta, se observaron dos tendencias durante la etapa de congelación, en las cuales la penetración del frío ocurrió así: primero, el calor salió lentamente por la presencia de una barrera a la conducción de calor y por la considerable proporción de agua líquida presente en el alimento, la cual es menos conductora que el hielo. Posteriormente, se dio la conducción rápida de calor al interior del alimento frío, que contenía una capa considerable de cristales de hielo [10].

La temperatura en la cual los cristales de soluto se encontraron en equilibrio con el agua líquida y el hielo se conoce como la temperatura eutéctica, y correspondió al máximo grado de solidificación. A partir de este momento no se retiró más calor 
latente, por lo que se observó un cambio en la pendiente de la curva [11][12].

En la Fig. 2, el final de la congelación se debió a la cristalización del agua libre, mientras que en el proceso criogénico este punto correspondió al cambio de estado del agua libre, el agua ligada, y otros macro nutrientes como los lípidos [14], [15].

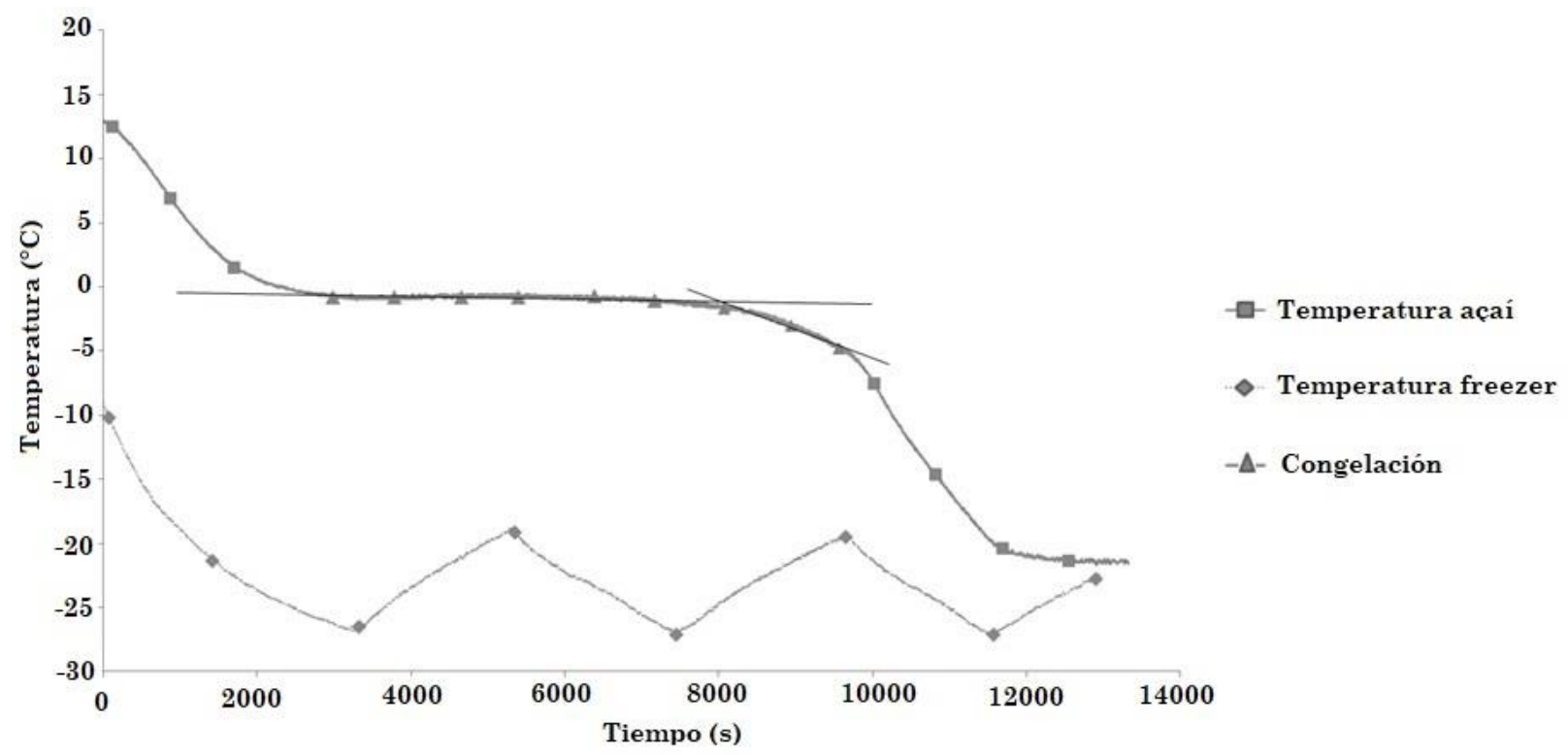

Fig. 2. Curva para la pulpa de açaí en congelador convencional. Temperatura del aire de $-22{ }^{\circ} \mathrm{C}$. Fuente: autores.

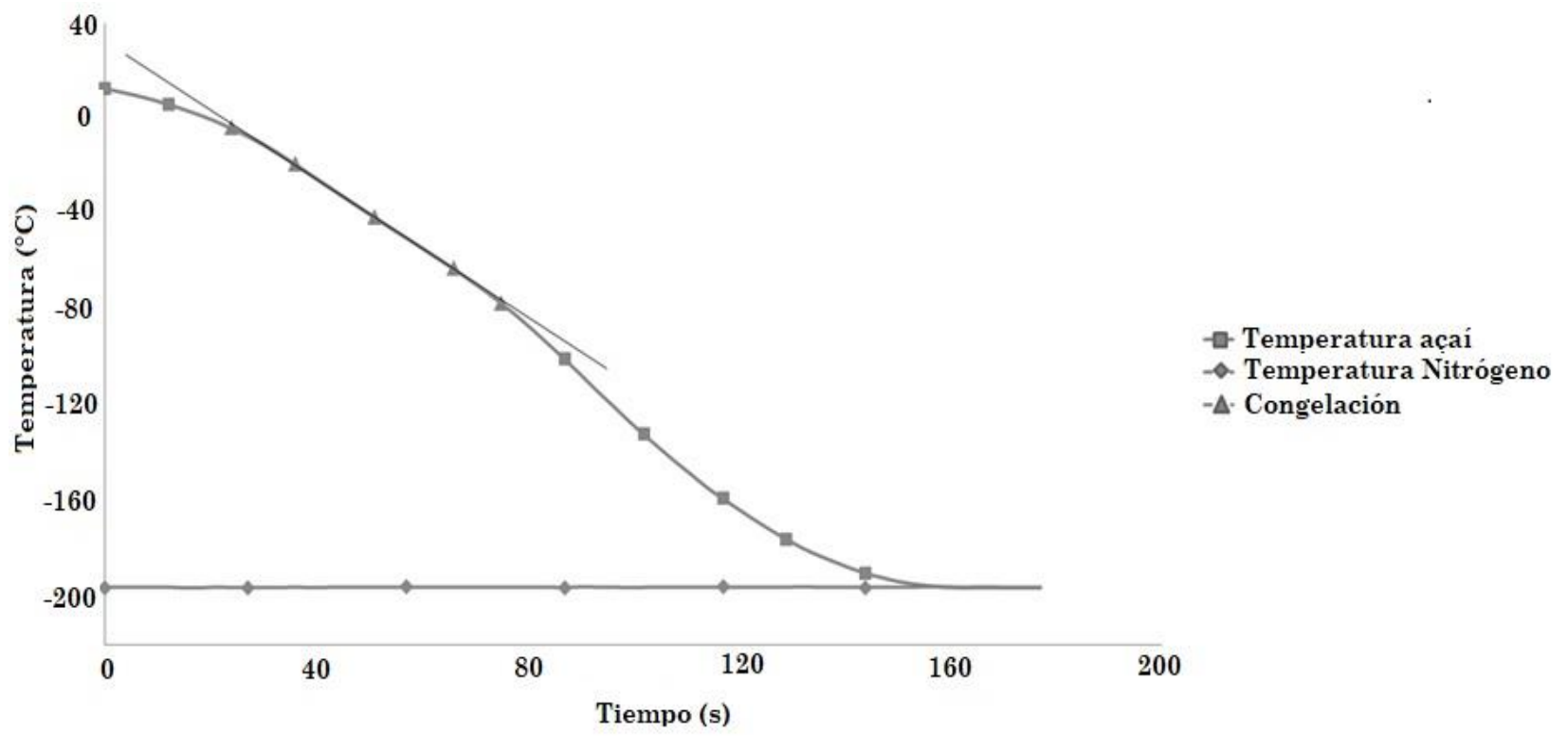

Fig. 3. Curva de congelación criogénica para la pulpa, con temperatura fría de $-196{ }^{\circ} \mathrm{C}$. Fuente: autores. 


\subsection{Temperatura inicial de congelación}

Para las frutas y vegetales, una variable fundamental en el estudio de su industrialización es la temperatura inicial de congelación, ya que corresponde normalmente al valor empleado para su almacenamiento en cámaras frías [26], [27]. En la Tabla 2 se presentan los resultados para la pulpa de açaí.

Las temperaturas iniciales de congelación de la pulpa se encontraron dentro del rango reportado por la ASHRAE [26] para algunas frutas. Además, los valores por debajo de $0{ }^{\circ} \mathrm{C}$ se explican por el descenso del punto crioscópico, una propiedad coligativa de las soluciones, ocasionada por la presencia o adición de solutos no volátiles en un sistema alimentario. Como la solución disminuyó su presión de vapor, el sólido igualó dicha presión, y la solidificación tuvo lugar a una temperatura más baja [9].

[28] y [29] encontraron temperaturas iniciales de congelación, para el limón tahiti y la acerola (Malpighia emarginata), cercanas a $-1{ }^{\circ} \mathrm{C}$. Estas son similares a las reportadas por la presente investigación, bajo el esquema de congelación convencional.

Fue evidente la diferencia entre el punto inicial de congelación del proceso rápido y el convencional. La divergencia entre estas temperaturas se debió a las condiciones en las cuales se efectuó cada experimento, puesto que la presión de trabajo del sistema criogénico fue mayor que la presión normal. La pulpa de açaí se sometió a la sumatoria de la presión atmosférica local, la presión ejercida por la columna de nitrógeno líquido y la presión ejercida por el gas. Para el agua pura, y de forma homóloga para las soluciones acuosas, la temperatura de fusión depende de la presión del sistema. Con un aumento de la presión, la temperatura de solidificación disminuye [30].

Se pudo concluir que la ecuación derivada de la ley de Raoult es útil únicamente para soluciones a presión atmosférica, pues incluye el término de la constante crioscópica del agua, la cual está determinada para el equilibrio sólido líquido en condiciones normales [19], [30].

\subsection{Tiempos de congelación}

Se analizaron los tiempos efectivos de congelación, para pulpa de açaí con temperatura inicial de $13{ }^{\circ} \mathrm{C}$. Para efectos de ingeniería, no es útil contar únicamente con los tiempos nominales de congelación, pues debe considerarse el gasto energético que involucra retirar el calor sensible de los productos [17].

Tabla 2. Temperaturas iniciales de congelación para pulpa de açaí. Fuente: autores

\begin{tabular}{l|lcc}
\hline \multicolumn{4}{c}{ Temperatura inicial de congelación de la pulpa } \\
\hline Fuente & \multicolumn{2}{c}{ Inicio de la congelación * $\left.\mathbf{(}^{\circ} \mathbf{C}\right)$} \\
\hline & $-0,62$ & $\mathbf{D}$ & 0,03 \\
Ley de Raoult & $-2,91$ & $\mathbf{b}$ & 0,86 \\
Real criogénico & $-0,64$ & $\mathbf{a}$ & 0,02 \\
Real convencional & \multicolumn{3}{c}{1,24} \\
\hline D.M.S &
\end{tabular}

* Valores promedio (+/- desviación estándar DE), $n=3$ y significancia $\alpha=0,05$. Medias seguidas de una misma letra, para cada temperatura inicial de congelación, no difieren entre sí por el test de Tukey. 


\subsubsection{Tiempos de congelación}

En la Tabla 3 se presentan los tiempos obtenidos para congelación convencional, siendo los modelos de Pham y Nagaoka los que mejor describieron la cinética del proceso.

Las ecuaciones de Pham y Nagaoka ofrecieron la ventaja de estimar la entalpía del proceso combinado de refrigeración y congelación, eliminando el error proveniente del cálculo del tiempo de enfriamiento mediante métodos clásicos [9].

Las imprecisiones del modelo de Plank se deben a las suposiciones sobre las cuales se formuló; entre ellas, se asumió constante la temperatura de congelación del producto, lo cual es válido únicamente para sustancias puras [8], [31]. En comparación con este modelo, la expresiones de Pham y Nagaoka tienen en cuenta las diferentes temperaturas por las que pasa el producto durante el proceso, con el fin de estimar las entalpías combinadas y la fracción de agua congelada [11], [22].

El modelo de Pham presentó el menor error respecto a datos reales. Sin embargo, el valor calculado para el factor de forma $(E=3,57)$ fue mayor que el establecido por el autor, denotando que no se trata de una geometría plana y que la transferencia de calor por conducción ocurrió simultáneamente en las tres direcciones
[19]. Este fenómeno afecta la exactitud de los resultados arrojados por el modelo, pues se encuentra basado en una metodología que considera el intercambio de energía unidireccional. Se recomienda, para futuros estudios, garantizar la geometría de la placa plana de fruta. Así, se asegura que la pérdida de calor prima en un sentido, de mayor importancia en comparación con las dos direcciones restantes [8].

Los resultados obtenidos en la presente investigación son similares a los reportados por Soares, et al. [32], donde la ecuación de Pham ofreció el mejor ajuste en la predicción de tiempos de congelación. El error promedio, en el caso de los autores, fue de 1,4\% para la conservación de mangaba (Hancornia Speciosa Gomes).

\subsubsection{Congelación con nitrógeno líquido}

La Tabla 4 presenta los tiempos de congelación rápida para la pulpa. La fracción de agua congelada en el producto se asumió igual a 1; la temperatura del nitrógeno líquido asegura la solidificación del agua ligada del producto [15]. La predicción de los tiempos de proceso utilizando la ecuación de Plank tuvo una notable mejoría, puesto que en este caso el agua libre y ligada se congelaron, y se tuvo una remoción completa de calor latente en el agua del alimento.

Tabla 3. Tiempos de congelación convencionales para la pulpa de açaí. Fuente: autores. Tiempo de congelación convencional para la pulpa de açaí

\begin{tabular}{l|cccc}
\hline Fuente & Tiempo de congelación * (min) \\
& Media & DE & Error \\
\hline Ecuación de Plank & 230,57 & $\mathbf{a}$ & 3,23 & $50,03 \%$ \\
Ecuación de Nagaoka & 151,06 & $\mathbf{b}$ & 4,81 & $1,71 \%$ \\
Ecuación de Pham & 154,50 & $\mathbf{b}$ & 5,17 & $0,54 \%$ \\
Real & 153,68 & $\mathbf{b}$ & 6,42 & - \\
\hline D.M.S & \multicolumn{4}{c}{13,164} \\
* Valores promedio (+/- desviación estándar DE), n=3 y a=0,05. Medias seguidas de una \\
misma letra, para cada tiempo de congelación convencional, no difieren entre sí por test \\
de Tukey.
\end{tabular}


Evaluación de los parámetros del proceso de congelación para la pulpa de Açaí

Tabla 4. Resultados de los tiempos de congelación criogénicos para la pulpa de açaí in natura. Fuente: autores.

\begin{tabular}{|c|c|c|c|c|}
\hline \multicolumn{5}{|c|}{ Tiempo de congelación criogénico para la pulpa } \\
\hline \multirow[t]{2}{*}{ Fuente } & \multicolumn{4}{|c|}{$\begin{array}{c}\text { Tiempo de congelación * } \\
\text { (s) }\end{array}$} \\
\hline & Media & & DE & Error \\
\hline Ecuación de Plank & 120,31 & $\mathbf{a}$ & 0,47 & $19,64 \%$ \\
\hline Ecuación de Nagaoka & 107,41 & $\mathbf{a}$ & 2,22 & $6,81 \%$ \\
\hline Ecuación de Pham & 73,82 & b & 1,31 & $26,59 \%$ \\
\hline Real & 100,56 & $\mathbf{a}$ & 17,90 & - \\
\hline
\end{tabular}

* Valores promedio (+/- desviación estándar DE), n=3 y significancia de 0,05. Medias seguidas de una misma letra, para tiempo de congelación criogénico, no difieren entre sí por el test de Tukey.

Tabla 5. Temperaturas finales de congelación de la pulpa de açaí, en tratamiento rápido y lento. Fuente: autores.

\begin{tabular}{lcccc}
\hline \multicolumn{3}{c}{ Temperaturas finales de congelación de la pulpa de açaí } \\
\hline & Congelación & \multicolumn{3}{c}{ Congelación } \\
& $\begin{array}{c}\text { convencional } \\
\text { Media }\end{array}$ & DE & Criogénica \\
& Media & DE \\
\hline $\mathrm{T}^{\circ}$ final de congelación $\left({ }^{\circ} \mathrm{C}\right)$ & $-6,50$ & 0,87 & $-69,94$ & 7,77 \\
\hline
\end{tabular}

Tabla 6. Cinéticas de congelación para cada uno de los esquemas de operación.

\begin{tabular}{cccc}
\multicolumn{4}{c}{ Fuente: autores. } \\
\hline \multicolumn{2}{c}{ Tasa de congelación $(\mathbf{c m} / \mathbf{h})$} & Velocidad de congelación $\left({ }^{\circ} \mathbf{C} / \mathbf{s}\right)$ \\
\hline $\begin{array}{c}\text { Congelación } \\
\text { convencional }\end{array}$ & $\begin{array}{c}\text { Congelación } \\
\text { criogénica }\end{array}$ & $\begin{array}{c}\text { Congelación } \\
\text { convencional }\end{array}$ & $\begin{array}{c}\text { Congelación } \\
\text { criogénica }\end{array}$ \\
\hline 0,78 & $\begin{array}{c}71,60 \\
\text { Ultra- } \\
\text { Semi - rápido }\end{array}$ & 0,006 & 0,67 \\
& rápido & Lento & Rápido \\
\hline
\end{tabular}

Además, debido a que el agua libre se congeló de forma inmediata, se pudo asumir que el cambio de fase ocurrió a temperatura constante [17]. Machado y Vélez [31] encontraron que este modelo fue el que mejor se adaptó a la congelación de diferentes alimentos mexicanos, como chile poblano, masa de tortilla, nopal y queso.

Nuevamente, el modelo de Nagaoka presentó el mayor grado de ajuste con los datos experimentales, gracias a los factores de corrección aplicados a los tiempos de pre enfriamiento del alimento.

En cuanto al modelo de Pham, con la geometría utilizada, el factor de forma $(E=$ 4,53) no permitió estimar el tiempo de congelación con exactitud. Soares, et al. [33] sugieren su aplicación en fenómenos con transferencia de calor unidireccional, cuando la forma de una placa de alimento no se asemeje a la de una esfera absoluta. Además, el ajuste de la ecuación a los datos experimentales se ve afectado por la alta velocidad de enfriamiento [19], [26].

Soares, et al. [33] encontraron un mayor ajuste del modelo de Plank, en comparación con la ecuación de Pham, para la congelación de geometrías no planas. A mayor grosor de la placa, incrementó el error en la propuesta de Pham. Esto se asemeja a los resultados hallados en el presente estudio. 


\subsection{Otros resultados}

\subsubsection{Temperatura final de congelación}

En la Tabla 5 se observan las temperaturas finales de congelación encontradas para la pulpa de açaí, en las dos velocidades de operación.

La gran cantidad de agua presente en el alimento, y de forma general en las frutas y pulpas, explica por qué la depresión del punto de congelamiento no fue muy grande en el caso convencional, exhibiendo un comportamiento parecido al del agua pura [27]. Para la congelación criogénica, se observó que el punto de congelación del agua ligada es mucho menor, debido a que existe una alta barrera a la transferencia de calor por conducción al interior del alimento, debida a la presencia de espacios vacíos, al contenido de otros macro componentes (lípidos, proteínas y carbohidratos), y a la existencia de un agua líquida de baja conductividad térmica [11], [17].

\subsubsection{Temperatura final de congelación}

Las tasas y velocidades de congelación obtenidas se relacionan en la Tabla 6. La congelación con nitrógeno líquido se clasificó como rápida, mientras que el congelamiento con aire se clasificó como un proceso de baja o media velocidad [10], [19].

Souza, et al. [34] presentan, a partir de $\mathrm{su}$ investigación con maracuyá, tasas y velocidades de congelación convencional similares a las encontradas en esta investigación. Para la congelación de frutas y pulpas se utilizan normalmente configuraciones lentas, debido a que estos productos poseen puntos de congelación altos y su producción resulta ser así más económica [27].

\section{CONCLUSIONES}

Para las dos velocidades de congelación, las temperaturas iniciales de cambio de fase fueron diferentes, concluyéndose que el modelo de predicción basado en la Ley de Raoult es válido únicamente a presiones normales. Se confirma la reducción del punto inicial de congelación cuando el sistema se encuentra sometido a presiones mayores que las atmosféricas, como es el caso de la inmersión criogénica.

En el congelamiento lento, los modelos de Pham y Nagaoka son los que mejor describen la variación de la temperatura en función del tiempo, mientras que la ecuación de Nagaoka se adapta a las condiciones de congelación rápida. La predicción de los tiempos se ve afectada, además de las variables analizadas en este estudio, por la geometría del alimento, su composición, la presión del sistema y el esquema tecnológico (convencional o criogénico). El valor del coeficiente convectivo de transferencia de calor influye especialmente en los casos con velocidad de operación baja.

La temperatura final de congelación, determinada durante la operación rápida, corresponde al punto donde cambian de fase todos los componentes presentes en la pulpa de açaí. El diferencial de temperatura entre el producto y el medio frío son lo suficientemente grandes como para alcanzar los criterios de solidificación del agua ligada, los lípidos, y las disoluciones de carbohidratos y proteínas presentes en el alimento.

Por medio de este trabajo, se muestra la importancia de las investigaciones en busca de datos para la ciencia y tecnología de los alimentos, gracias a que facilitan la operación y el control eficaz de los procesos productivos.

Más aún, por medio del modelamiento matemático, se favorece el diseño y la puesta en marcha de las diferentes operaciones; conociendo, de manera preliminar, los periodos de tiempo y las 
condiciones de calidad de los alimentos que se someterán a cualquier actividad de conservación. A esto se refiere la predicción en ingeniería.

En el caso específico del açaí, el avance en esta área tiene gran importancia dadas sus altas tasas de producción, comercialización y consumo en el Brasil. Las principales propuestas de conservación de este alimento son el enfriamiento y la congelación. La implementación de un buen esquema tecnológico, la selección de una correcta temperatura de congelación y la predicción de los tiempos de proceso son primordiales para evitar que la pulpa de açaí, o de cualquier otra fruta, se someta a tratamientos excesivos que ocasionen el deterioro de los atributos organolépticos y nutricionales de la matriz vegetal.

Para la pulpa de açaí se sugiere, entonces, un proceso de congelación convencional que no alcance una temperatura inferior a $-6,5{ }^{\circ} \mathrm{C}$. En el caso criogénico, la congelación se da casi de forma inmediata, siendo total a una temperatura cercana a $-70{ }^{\circ} \mathrm{C}$. Los tiempos para cada operación, respectivamente, no deben superar los 154 y 1,7 min.

\section{AGRADECIMIENTOS}

A la Universidad Federal de Viçosa Brasil, a la Universidad de Caldas Colombia, a la Universidad Católica Luis Amigó - Colombia, quienes apoyaron el desarrollo y la divulgación del presente proyecto de investigación.

\section{REFERENCIAS}

[1] CONAB, “Análise mensal: açaí (fruto).” CONAB - Companhia Nacional de Abastecimento do Brasil, Brasilia, Brasil, Jan. 2019.

[2] P. A. A. C. de Oliveira, I. G. da Silva, M. L. de Souza, C. M. Furtado, and R. F. da Silva, "In natura açaí beverage: quality, pasteurization and acidification," Food Sci.
Technol., vol. 31, no. 2, pp. 502-507, Jun. 2011. https://doi.org/10.1590/S010120612011000200035

[3] E. M. da S. Menezes, A. T. Torres, and A. U. Sabaa Srur, "Valor nutricional da polpa de açaí (Euterpe oleracea Mart) liofilizada," Acta Amaz., vol. 38, no. 2, pp. 311-316, 2008. https://doi.org/10.1590/S004459672008000200014

[4] A. G. Asuero, R. Fett, and E. M. Kuskoski, "Antocianos, un grupo de pigmentos naturales. Aislamiento, identificación y propiedades," Aliment. Rev. Tecnol. e Hig. los Aliment., vol. 339, pp. 61-74, 2002.

[5] A. K. O. Homma et al., Sistema de produção do açaí: Sistemas de produção No. 4, 2nd ed. Belém, Brasil: Embrapa - Empresa Brasileira de Pesquisa Agropecuaria, Amazonia Oriental, 2005.

[6] N. Sanabria and E. Sangronis, "Caracterización del açaí o manaca (Euterpe olerácea), un fruto del amazonas," Arch. Latinoam. Nutr., vol. 57, no. 1, pp. 94-98, Nov. 2007.

[7] D. Pompeu, V. Barata, and H. Rogez, "Impacto da refrigeração sobre as variáveis de qualidade dos frutos do açaizeiro (Euterpe olerácea)," Aliment. e Nutr. Araraquara, vol. 20, no. 1, pp. 141-148, Jan. 2009.

[8] C. J. Geankoplis, Procesos de transporte $y$ operaciones unitarias, 3rd ed. Ciudad de México, México: Compañia Editorial Continental, 1998.

[9] J. A. Barreiro and A. J. Sandoval, Operaciones de conservación de alimentos por bajas temperaturas, 1st ed. Caracas, Venezuela: Equinoccio, 2006.

[10] K. Cháves, L. Marques, and J. Freire, "Determinação das curvas de congelamento em freezer convencional e Nitrogênio líquido da pimenta dedo de moça," in VIII Congresso Brasileiro de engenharia quimica e iniciação científica, Minas Gerais, 2009.

[11] C. E. Orrego, Congelación y liofilización de alimentos. Manizales, Colombia: Editorial Universidad Nacional de Colombia, 2008.

[12] E. Umaña, "Conservación de alimentos por frio: Refrigeración y congelamiento." Fiagro y Fusades proinnova, San Salvador, El Salvador, 2007.

[13] M. C. Erickson y Y. C. Hung, Freezing systems: Quality in Frozen Foods. Boston, MA: Springer US, 1997.

https://doi.org/10.1007/978-1-4615-5975-7

[14] J. V. Neckel and C. V. Mariani, "Modelagem do congelamento da Beterraba," Asoc. Argentina Mecánica Comput., vol. 29, pp. 5729-5740, Nov. 2010.

[15] S. Badui, Química de alimentos, 4th ed. Ciudad de México, México: Pearson 
Ecucación, pp. 738. 2006.

[16] M. S. Araújo, M. E. Braga, and M. E. Moreira, "Cinética de congelamento de polpa de acerola a baixas temperaturas," Rev. Bras. Prod. agroindustriais, vol. 2, no. 1, pp. 27-34, 2000. http://dx.doi.org/10.15871/15178595/rbpa.v2n1p27-34

[17] P. J. Fellows, Tecnología do processamento de alimentos principios de practica, 2nd ed. Porto Alegre, Brasil: Artmed, 2006.

[18] H. P. Gurney and J. Lurie, "Charts for Estimating Temperature Distributions in Heating or Cooling Solid Shapes.," Ind. Eng. Chem., vol. 15, no. 11, pp. 1170-1172, Nov. 1923. https://doi.org/10.1021/ie50167a028

[19] C. E. Orrego, Procesamiento de alimentos. Manizales, Colombia: Editorial Universidad Nacional de Colombia, pp. 322. 2003.

[20] S. Arias, A. M. Ceballos, and L. F. Gutiérrez, "Estudio de composición centesimal y propiedades termo-físicas para la pulpa de açaí," Vitae, vol. 23, no. 1, pp. 140-144, 2016.

[21] C. S. Chen, "Thermodynamic Analysis of the Freezing and Thawing of Foods: Enthalpy and Apparent Specific Heat," J. Food Sci., vol. 50, no. 4, pp. 1158-1162, Jul. 1985. https://doi.org/10.1111/j.1365-

2621.1985.tb13034.x

[22] H. A. Jiménez, L. Chaires, and M. A. Pérez, "Prediction of Freezing Time and Evaluation of the Effect of Frozen Storage on Textural Properties of White Trout Fillets," in Food Sci. Technol. Res., 2013, vol. 19, suplemento. 3 pp. 375-379.

https://doi.org/10.3136/fstr.19.375

[23] Q. T. Pham, "Simplified equation for predicting the freezing time of foodstuffs," Int. J. Food Sci. Technol., vol. 21, no. 2, pp. 209-219, Oct. 1986.

https://doi.org/10.1111/j.1365-

2621.1986.tb00442.x

[24] D. Cleland and K. Valentas, Handbook of food engineering practice Chapter 3 Prediction of freezing time and design of food freezers. Boca Ratón, USA: CRC Press, pp. 698. 1997.

[25] M. López-Leiva and B. Hallström, "The original Plank equation and its use in the development of food freezing rate predictions," J. Food Eng., vol. 58, no. 3, pp. 267-275, Jul. 2003.

https://doi.org/10.1016/S0260-8774(02)00385-
0

[26] Ashrae American of Heating, 1998 Ashrae Handbook: refrigeration: systems and applications. Atlanta, USA: American Society of Heating Refrigerating and Air Conditioning Engineers, 1990.

[27] J. E. Lozano, Fruit Manufacturing: Scientific Basis, Engineering Properties, and Deteriorative Reactions of Technological Importance. New York: Springer US, 2006. https://doi.org/10.1007/978-0-387-30616-2

[28] K. D. Brito, L. S. C. Oliveira, A. G. B. de Lima, A. M. Santiago, and D. E. G. Alves, "Avaliação do processo físico de congelamento da polpa de limão tahiti (citrus latifólia tanaka)," in Anais do XX Congresso Brasileiro de Engenharia Química, 2015. pp. 3591-3598. https://doi.org/10.5151/chemengcobeq2014-0488-25253-144251

[29] C. G. Pereira, "Propriedades termofísica e comportamento reológico de polpa de acerola em diferentes concentrações e temperaturas," Universidade Federal de Lavras, Lavras, Brasil, 2013.

[30] J. M. Rodríguez and R. Marín, Fisicoquímica de aguas, 1st ed. Madrid, España: Diaz de Santos, 1999.

[31] K. M. Machado and J. F. Vélez, "Estudio de propiedades físicas de alimentos mexicanos durante la congelación y el almacenamiento congelado," Rev. Mex. Ing. química, vol. 7, no. 1, pp. 41-54, Apr. 2008.

[32] D. S. C Soares, J. T. S. Santos, A. F. P. Campos, F. S. C. Costa, T. P. Nunes, and A. M. O. Júnior, "Avaliação do tempo de congelamento da mangaba (Hancornia Speciosa Gomes) em ultrafreezer através dos modelos matemáticos de Planck e Pham," Sci. Plena, vol. 8, no. 4, pp. 1-6, 2012.

[33] D. S. C Soares, G. F. Silva, T. C. S. Silva, T. P. Nunes, and A. M. Oliveira Júnior, "Halfripe mangaba (Hancornia Speciosa Gomes) freezing kinetics with different diameters," Chem. Eng. Trans., vol. 29, pp. 1411-1416, 2012. https://doi.org/10.3303/CET1229236

[34] D. S. Souza, J. D. R. Pimentel, J. A. B. dos Santos, and N. Narain, "Study on the freezing kinetics of passion fruit pulp," Acta Hortic., vol. 1198, no. 1198, pp. 263-264, Apr. 2018.

https://doi.org/10.17660/ActaHortic.2018.119 8.44 